DOE/ER/13590--12

DE93 008211

\title{
IONIZATION PROBES OF MOLECULAR STRUCTURE AND CHEMISTRY
}

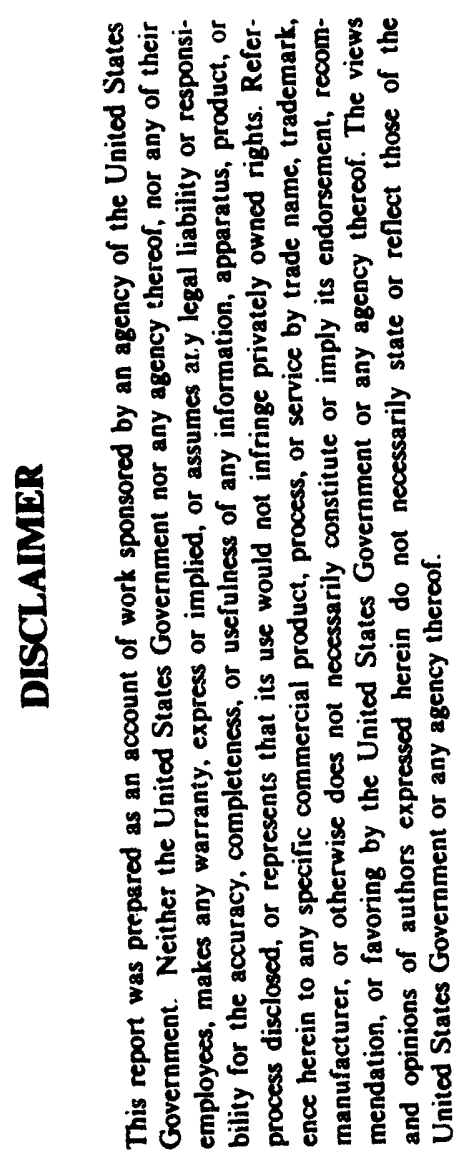

\author{
Progress Report \\ for Period January 15, 1992-January 14, 1993
}

Philip M. Johnson

State University of New York

Stony Brook, NY 11794

January 1993

Prepared for

THE U.S. DEPARTMENT OF ENERGY

AGREEMENT NO. DE-FG02-86ER13590

\section{MASTER}




\section{INTRODUCTION}

We have continued the development of laser ionization tools toward the study of the spectroscopy and dynamics of molecules relevant to combustion processes. When high intensity laser beams interact with any physical system composed of electrons and nuclei, an almost inevitable result of the high electric field strengths in the light beam is that electrons are separated from the nuclei. This ionization, when carried out in a controlled way, can provide a great deal of information about the molecular system, including energies and dynamics of electronic states, and can provide a fingerprint for the detection of an atom or molecule.

\section{IONIZATION VERSUS DISSOCIATION IN $\mathrm{CO}_{2}$}

One of the molecules we have spent considerable effort on is carbon dioxide. Besides its obvious relevance in combustion, it presents a great challenge to spectroscopy because of its propensity toward dissociation in all of its excited states. Multiphoton ionization spectroscopy is usually not applicable to the study of dissociating molecules because the dissociation competes effectively with ionization, resulting in no signal. However, with high enough laser fluence, ionization can compete with dissociation in the longer lived states, exposing them for study from the continuous spectral background resulting from rapidly dissociating states. The intense laser field produces effects not seen in ordinary spectroscopy, and we have been studying the various spectroscopic and photophysical effects found through the multiphoton ionization and multiphoton photoelectron spectra.

Photoelectron spectroscopy is a very useful technique for finding out about properties of both neutral molecules and ions. For carbon dioxide it provides a means of determining photoionization and photodissociation mechanisms. Our previous studies on carbon dioxide have elucidated some of its multiphoton spectroscopy and discovered several fascinating photophysical processes. One of the more intriguing of these in terms of its possibilities concerning the detailed understanding of photodissociation is the appearance of long non-Franck-Condon progressions in the multiphoton photoelectron spectra. These appear to be indicative of some sort of ionization during the process of dissociation, and there is a potential to be able to learn about the details of dissociation. In the three-photon resonant four-photon ionization process, ionization could be competing with dissociation at either the three-photon or the four-photon level. For the former, a bound state would be predissociating, creating a wave packet which could be ionized at various points along the reaction coordinate. The photoelectron spectrum would be intensity dependent because higher laser fields would promote ionization before the wave packet has a chance to travel very far. At the four-photon level, excitation can be directly to a neutral dissociation continuum where the molecule can autoionize while in the process of dissociation. The photoelectron spectrum would not be intensity dependent.

We have measured the intensity dependence of the vibrational structure seen in the photoelectron spectra over almost a factor of two in laser intensity and found no changes, providing evidence that the multiphoton ionization of $\mathrm{CO}_{2}$ through its Rydberg states proceeds through autoionization of bound, neutral dissociative states at the four photon level. This may be explained by a propensity toward excitation of core electrons 
to antibonding orbitals in preference to excitation of a Rydberg electron which is decoupled from the core.

\section{HIGH RESOLUTION VIBRATIONAL SPECTROSCOPY OF CATIONS}

Threshold ionization spectroscopy provides similar information to photoelectron spectroscopy in that it locates the energy levels of an ion but it does so by means of scanning a tunable photon source over the ionization continuum looking for ionization thresholds. A recently developed variant of threshold ionization spectroscopy, usually called ZEKE, has shown to be as useful as traditional photoelectron spectroscopy (where the kinetic energy of ejected electrons are measured) but with higher resolution and much better signal-to-noise when using standard laboratory lasers. It has recently been realized that the $\mathrm{ZEKE}$ or pulsed field ionization (PFI) method of measuring threshold ionization spectra is exploiting the field ionization of very high Rydberg states which exist just before each ionization threshold.

Aromatic molecules are of great importance in combustion research since they constitute a significant fraction of unleaded gasoline. We chose to develop our capabilities in threshold ionization spectroscopy using aromatic molecules because of their importance and because their electronic structure allows a pump-probe type of excitation scheme which avoids the use of vacuum ultraviolet laser beams. Among aromatics, the diazines are noted for their small $S_{1}-T_{1}$ energy gap which gives them unique and interesting photophysical properties. Their electronic struciure is also interesting because lone-pair electrons in almost non-interacting orbitals produce an effect called "spontaneous symmetry breaking" in SCF calculations and may have implications in the structure of the molecule itself, particularly in the ion. We have studied the basic threshold ionization spectroscopy of diazines pyrazine and pyrimidine, acquiring high resolution vibrational spectra of the ground state ion while resonant with nine different vibrational levels of $S_{1}$.

In the last year we have explored the capabilities of ab initio force field calculations in understanding the ionic spectra. MP2/6-31G ${ }^{*}$ calculations of the vibrational frequencies of the neutral ground, $S_{1}$, and the ionic ground state have been compared with the experimental values, finding that certain vibrations of $S_{1}$ and the ion which engage in extensive vibronic coupling are not properly determined by the calculated force field. Most vibrational frequencies are accurately reproduced, however. Variations in the complexity of the threshold ionization spectra with the level of $S_{1}$ excitation indicate that internal vibrational relaxation is taking place at a very low energy in that state, possible involving vibronic interactions and mixing with the triplet manifold.

In the course of the experimental work on the azines we developed a new technique of detecting ionization thresholds which depends only upon measuring the ions produced by field ionization of high Rydberg states and therefore retains mass information. This mass analyzed threshold ionization spectroscopy (MATI) is done by exploiting the narrow kinetic energy distribution of the molecules in a supersonic beam and the fact that near a threshold ions can be separated from Rydbergs by a small electric field. Field ionized Rydbergs are made to arrive at the detector before any directly produced ions of the same mass, and gating on the earlier arriving species while scanning ionization wavelengths produces a threshold ionization spectrum of only the 
mass of interest. The viability of the scheme was demonstrated by producing the same spectrum for pyrazine by electron and ion detection.

Recently we have been working on the improvement of the MATI apparatus, particularly with respect to its mass resolution. The very low voltages necessary in the source region of this machine present a very great challenge to the design. To that end we have designed and built a much more sophisticated spectrometer which has space focussing and velocity focussing for the ions in a tandem geometry (Fig. 1). With this we have substantially improved the mass resolution of the MATI technique to a very usable form, as shown in the second figure where the peak labelled F.I. is from the field ionized Rydbergs. Further development is under way aimed toward enabling MATI to be used at higher temperatures, possibly even ambient, where thermal velocities are significant with respect to those provided by the low voltages of the source.

\section{COLLABORATIONS}

We have continued our collaborations with Trevor Sears and Michael White at Brookhaven National Laboratory.

In the diode laser infrared spectroscopy of radicals (with Sears) we have used a newly designed experimental system to investigate the $v_{2}$ fundamental of trans-HOCO and DOCO. For these important combustion radicals, the detailed rotational structure has been recorded and assigned. Significant perturbation were found which could be due to anharmonic interactions with combinations of lower frequency in-plane vibrations. This absorption spectrum will be useful in future studies of chemical reactions involving the radicals. Work on the ethyl radical continues.

We have also (with White) examined the vacuum ultraviolet photodissociation of three chlorofluoromethanes which are important in depletion of ozone in the upper atmosphere. This has been done by measuring the kinetic energy released in the chlorine atom fragments using multiphoton mass spectrometry. Several dissociation channels have been identified, depending upon the excited state being produced in the primary absorption process. Usually there exist both prompt and slow dissociation channels which are in kinetic competition.

\section{BUDGET}

The funding request is not changed from the one previously submitted at the time of renewal. There has been no change in any other funding for the principle investigator. 


\section{PUBLICATIONS}

Transient diode laser absorption spectroscopy of the $v_{2}$ fundamental of trans-HOCO and DOCO, T. Sears, W. Fawzy and P. Johnson, J. Chem. Phys. 97, 3996 (1992).

Resonance enhanced multiphoton ionization photoelectron spectra of $\mathrm{CO}_{2}$ III. Autoionization dominates direct ionization, D. Taylor and P. Johnson, J. Chem. Phys., to be published

Vibrations of pyrazine and its ion as studied by threshold ionization spectroscopy, L. Zhu and P. Johnson, submitted to J. Chem. Phys.

Mass analyzed threshold ionization: structural information for a mass spectrum and mass information for ionic spectroscopy, P. Johnson and L. Zhu, submitted to Intl. J. of Mass Spectrom. 


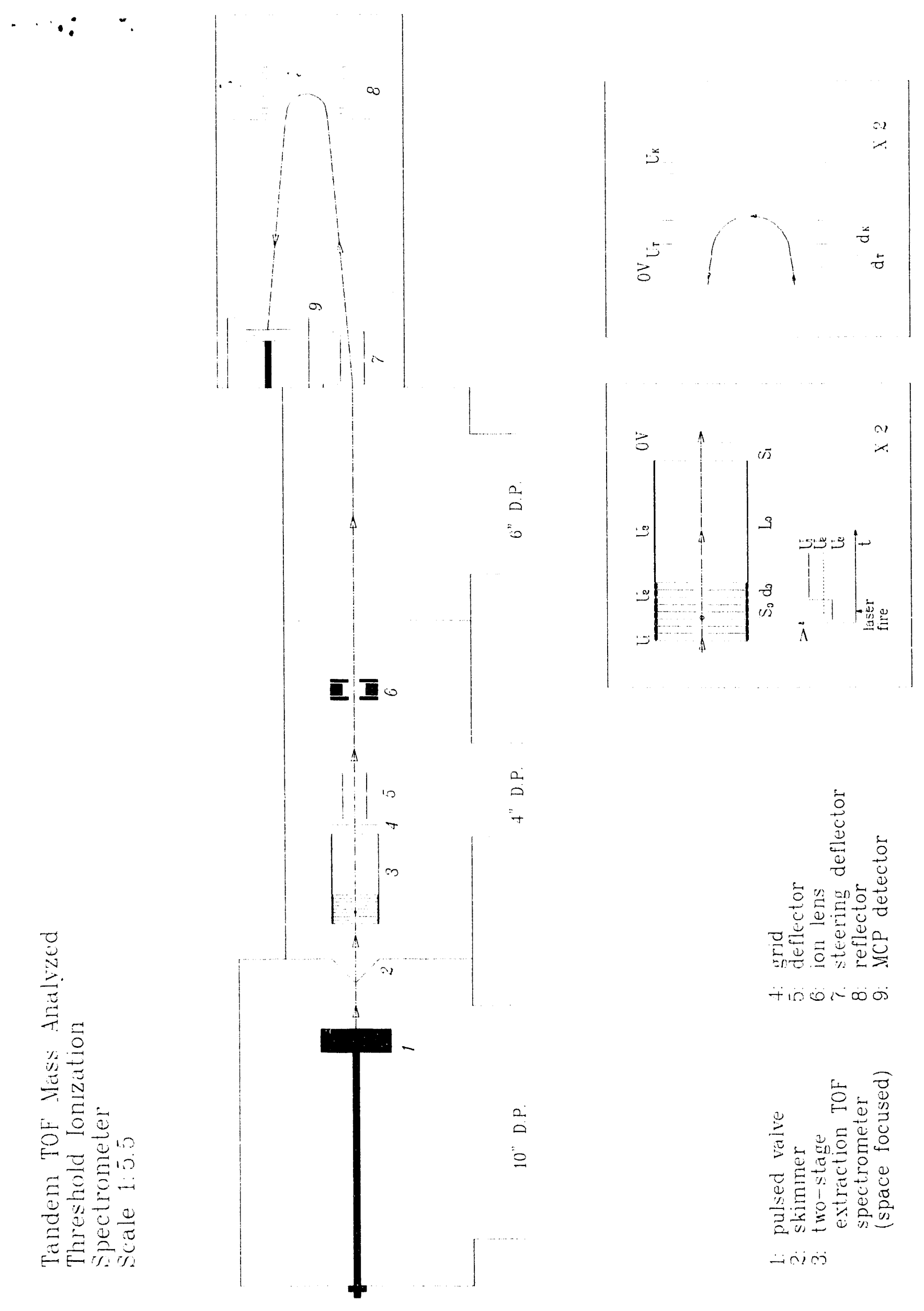




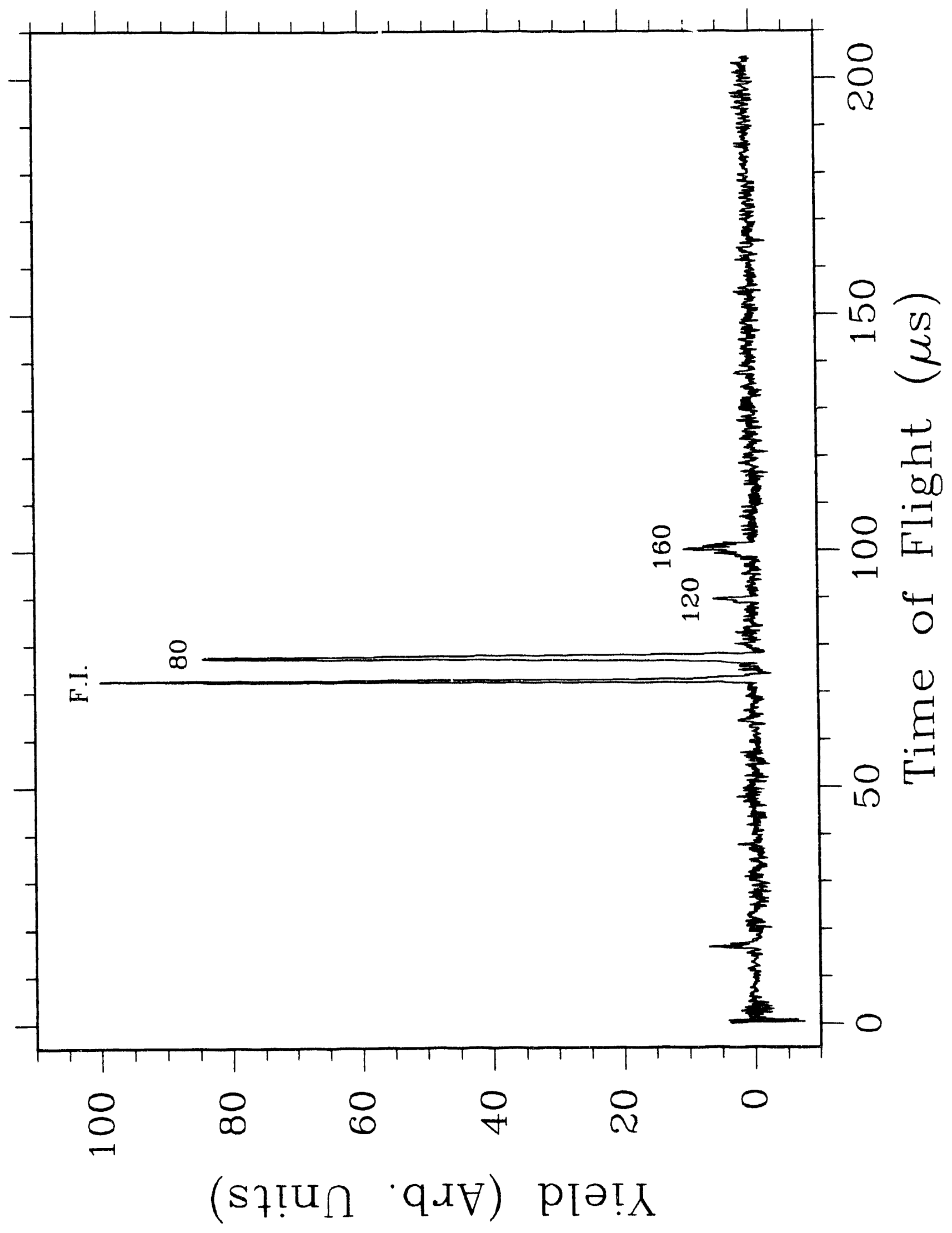



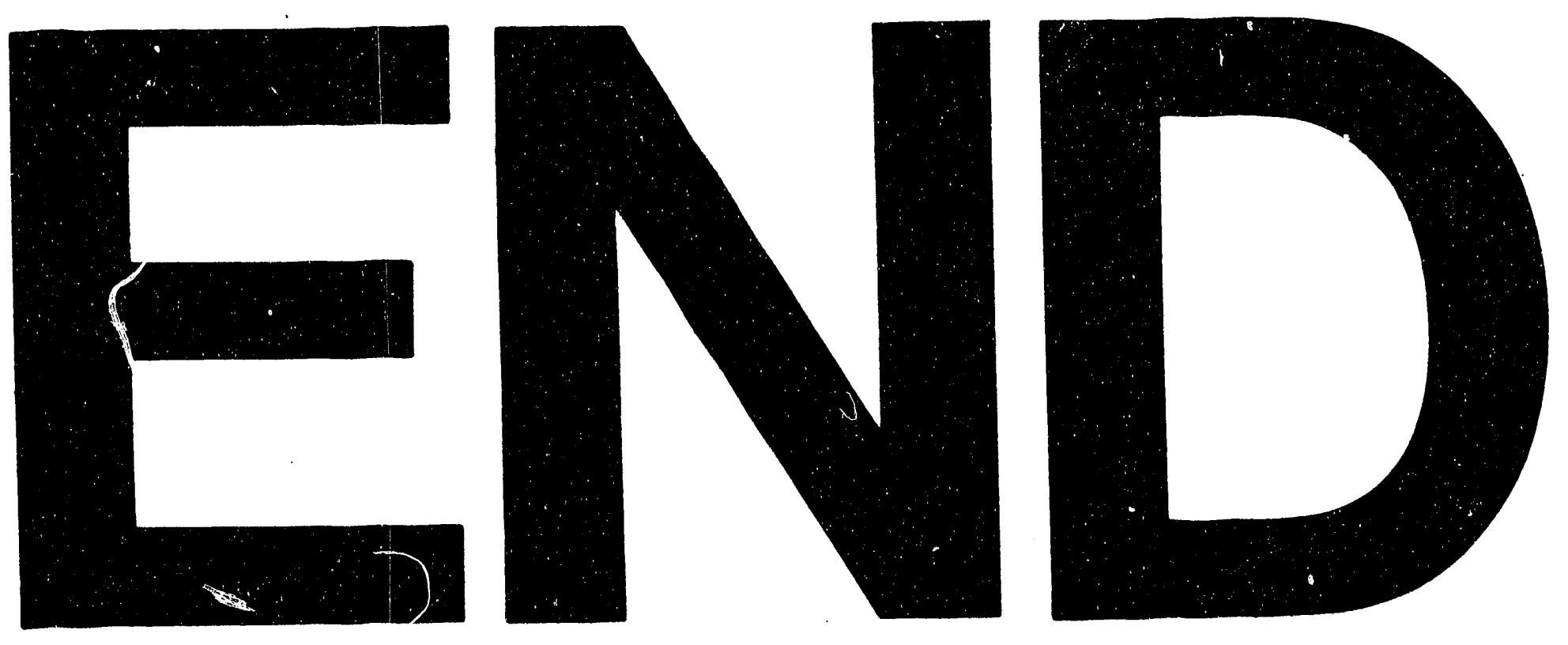

1
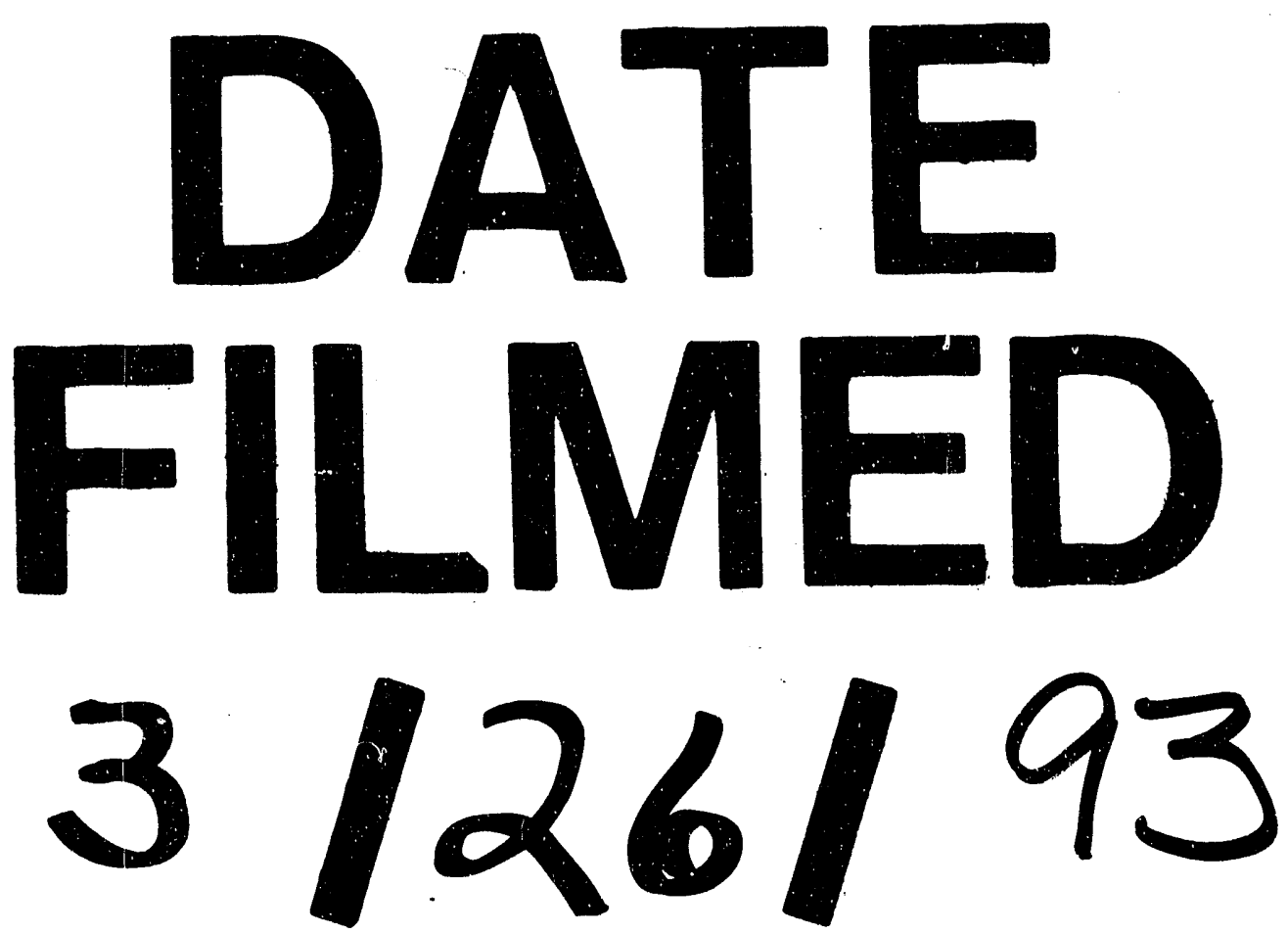
